Contents list available at JMCS

Journal of Mathematics and Computer Science

Journal Homepage: www.tjmcs.com

\title{
Application of the Exact Operational Matrices Based on the Bernstein Polynomials
}

\author{
K.Parand, Sayyed A. Kaviani \\ k_parand@sbu.ac.ir
}

\author{
Computer Sciences, Faculty of Mathematical Sciences \\ Shahid Beheshti University, Evin,Tehran 19839, Iran
}

\begin{abstract}
Article history:
Received March 2012

Accepted December 2012

Available online January 2013
\end{abstract}

\begin{abstract}
This paper aims to develop a new category of operational matrices. Exact operational matrices (EOMs) are matrices which integrate, differentiate and product the vector(s) of basis functions without any error. Some suggestions are offered to overcome the difficulties of this idea (including being forced to change the basis size and having more equations than unknown variables in the final system of algebraic equations). The proposed idea is implemented on the Bernstein basis functions. By both of the newly extracted Bernstein EOMs and ordinary operational matrices (OOMs) of the Bernstein functions, one linear and one nonlinear ODE is solved. Special attention is given to the comparison of numerical results obtained by the new algorithm with those found by OOMs.
\end{abstract}

Keywords: Exact Operational matrices, Bernstein polynomials, Bessel differential equation, Emden-Fowler equation.

AMS subject classification: 65M70, 65N35.

\section{Introduction}

In this paper, we introduce a new category of matrices by which some operations such as differentiation, integration and product can be done exactly. Also, we introduce some new matrices including Galerkin matrix, by which the resulting equations 
system of the Galerkin method is obtained. Hereon we introduce them briefly and leave the detailed explanation to be discussed in the main context.

\subsection{Operational matrices}

Orthogonal functions and polynomial series have received researchers' attention for solving various problems. The main role of this technique is to convert the problem to a system of algebraic equations, which is a considerable complexity reduction. This approach is based on the terms approximation of the polynomial series and the use of operational matrices for eliminating the integration, differentiation and products from the equation. For example, suppose the following truncated polynomial series and the known functions $f(x)$ and $g(x)$

$$
\begin{aligned}
& \Theta_{m}=\left\{\beta_{1}(x), \beta_{2}(x), \cdots, \beta_{m}(x)\right\} \\
& f(x)=\sum_{i=1}^{m} \kappa_{i} \beta_{i}(x)=k^{T} b_{m}(x) \\
& g(x)=\sum_{i=1}^{m} \lambda_{i} \beta_{i}(x)=l^{T} b_{m}(x),
\end{aligned}
$$

where

$$
\begin{aligned}
& b_{m}(x)=\left[\begin{array}{llll}
\beta_{1}(x) & \beta_{2}(x) & \cdots & \beta_{m}(x)
\end{array}\right]^{T}, \\
& k=\left[\begin{array}{llll}
\kappa_{1}(x) & \kappa_{2}(x) & \cdots & \kappa_{m}(x)
\end{array}\right]^{T}, \\
& l=\left[\begin{array}{llll}
\lambda_{1}(x) & \lambda_{2}(x) & \cdots & \lambda_{m}(x)
\end{array}\right]^{T} .
\end{aligned}
$$

We call the $b_{m}(x)$ the basis vector.Up to now, Researchers who have employed this approach, have been approximating the following expressions as

$$
\begin{aligned}
& \int_{0}^{x} f(x) d x=k^{T} \int_{0}^{x} b_{m(x)} \approx k^{T} P_{1} b_{m}(x) \\
& \frac{d}{d x} f(x)=k^{T} \frac{d}{d x} b_{m}(x) \approx k^{T} D_{1} b_{m}(x) \\
& f(x) g(x)=\left(k^{T} b_{m}(x)\right)\left(b_{m}(x)^{T} l\right) \approx k^{T} \widehat{L_{1}} b_{m}(x)
\end{aligned}
$$

where $P_{1}, D_{1}$ and $\widehat{L_{1}}$ are integration, differentiation and product matrices related to the basis $\Theta_{m}$. As it can be seen, both of $f(x)$ and $g(x)$ are in the $\operatorname{Span}\left(\Theta_{m}\right)$. It is quite probable that their integration or product do not remain in that space; but, to the best of our knowledge, in all of the previous researches they have been kept in the $\operatorname{Span}\left(\Theta_{m}\right)$. That is because the increase in the dimension causes the number of the algebraic system equations to be greater than the number of unknown variables. In this paper, a solution is presented for this problem.

Firstly, we present $P_{2}, D_{2}$ and $\widehat{L_{2}}$

$$
\begin{aligned}
& \int_{0}^{x} f(x) d x=k^{T} \int_{0}^{x} b_{m(x)}=k^{T} P_{2} b_{n_{1}}(x) \\
& \frac{d}{d x} f(x)=k^{T} \frac{d}{d x} b_{m}(x)=k^{T} D_{2} b_{n_{2}}(x) \\
& f(x) g(x)=\left(k^{T} b_{m}(x)\right)\left(b_{m}(x)^{T} l\right)=k^{T} \widehat{L_{2}} b_{n_{3}}(x) .
\end{aligned}
$$


As it can be seen, all of the approximations have been removed and also the basis vector has been changed. $b_{n_{i}}(x)$ depends on the $b_{m}(x)$ and the respective operational matrix.

We apply these matrices into the equation and sum all of the terms together to reach the residual function. To be able to factor out a basis vector from all of the different $b_{n}(x)$-sized terms in the residual function, the matrix $E_{i, j}$ is introduced, by which:

$$
b_{i}(x)=E_{i, j} \cdot b_{j}(x) .
$$

Using this matrix, we can convert the basis vector existing in each term to the biggestsized $b_{N}(x)$. By factoring out the $b_{N}(x)$, we can write the residual function as $R_{N} b_{N}(x)$; so, to solve the problem, we should solve

$$
R_{N} b_{N}(x)=0 .
$$

$b_{N}(x)$ is a basis vector and its functions are linearly-independent; so we can solve the following equation instead:

$$
R_{N}=0
$$

To overcome the problems of solving a system with $N$-equations and $m$ unknown variables, we introduce the Galerkin matrix $Q_{N, m}$, which reduces the number of equations to $m$, based on the Galerkin method

$$
R_{N} Q_{N, m}=0
$$

In this paper, we call the newly introduced exact operational matrices EOMs and the ordinary operational matrices OOMs.

\subsection{The previous researches}

The idea of operational matrices is very general and applicable to dozens of bases. Lots of researchers have worked on OOMs, using miscellaneous bases; but, to the best of our knowledge, the author's research is the first study on the EOMs.

Razzaghi et al., in [1],[2] and[3] presented the integral and product operational matrices based on the Fourier, the Taylor and the shifted-Jacobi series. Doha[4] has derived the shifted Jacobi operational matrix of fractional derivatives which is applied together with the spectral Tau method for the numerical solution of dynamical systems. Recently, Yousefi et al. in [5][6][7][8][9] and[10] have presented Legendre wavelets and Bernstein operational matrices and used them to solve miscellaneous systems. Also, recently, in [11] a new shifted-Chebyshev operational matrix of fractional integration of arbitrary order is introduced and applied together with the spectral Tau method for solving linear fractional differential equations (FDEs). In [12],[13] and [14] the operational matrix form in Hybrid of block-pulse functions and another set of functions like Taylor series, Legendre and Chebyshev has been used for finding the solution of the various classes of dynamical systems. Lakestani[15] constructed the operational matrix of fractional derivative of order $\alpha$ in the Caputo sense using the linear B-spline functions. In [16], a general formulation for the ddimensional orthogonal functions and their derivative and product matrices has been presented. OOMs have been utilized together with the Tau method to reduce the solution of partial differential equations (PDEs) to the solution of a system of algebraic equations. The authors of [17] presented a modified form of the homotopy analysis method based on Chebyshev operational matrices. Recently in [18], a class of two-dimensional nonlinear Volterra's integral equations has been solved using 
operational matrices of Legendre polynomials. The operational matrices of integration and product together with the collocation points have been utilized to reduce the solution of the integral equation to the solution of a nonlinear algebraic equations system. [19] presented a new Legendre wavelet operational matrix of derivation. In [20] and[21], the one and two dimensional fractional equations were studied by using Legendre operational matrices.

\subsection{The article structure}

In section 2, the idea is implemented by the Bernstein polynomials; also, the integration, differentiation, product and Galerkin matrices alongside some new matrices are introduced. Section 3 analyses the mean error bound of the Bernsteinbased best approximation. In section 4, a zero order Bessel and an Emden-Fowler differential equation are solved by both EOM and OOM approaches to prove the validity of the method and also the superiority of EOMs over OOMs. Finally, the $5^{\text {th }}$ section has been devoted to the conclusion and new suggestions for future studies.

\section{Bernstein polynomials (B-polynomials)}

\subsection{Overview of B-polynomials}

The Bernstein polynomials (B-polynomials) [10], are some useful polynomials defined on[0,1]. The Bernstein polynomials of degree $n$ form a basis for the power polynomials of degree $n$ [22]. We can mention lots of their properties. They are continuous over the domain. They satisfy symmetry

$$
B_{i, n}(t)=B_{n-i, n}(1-t) \text {, }
$$

positivity

$$
\forall t \in[0,1]: B_{i, n}(t) \geq 0,
$$

normalization or unity of partition [22]

$$
\sum_{i=0}^{n} B_{i, n}(t)=1 .
$$

Also, $B_{i, n}(t)$ in which $i \notin\{0, n\}$ has a single unique local maximum of

$$
i^{i} n^{-n}(n-i)^{n-i}\left(\begin{array}{c}
n \\
i
\end{array}\right)
$$

occurring at $t=\frac{i}{n}$. All of the B-polynomial bases take 0 value at $x=0$ and $x=1$, except the first polynomial at $x=0$ and the last one at $x=1$, which are equal to 1 . It can provide flexibility applicable to impose boundary conditions at the end points of the interval.

We present the solution of linear and nonlinear differential equations as linear combinations of the Bernstein polynomials, $P(x)=\sum_{i=0}^{n} c_{i} B_{i, n}(x)$, and the coefficients $c_{i}$ are determined using the Galerkin method.

In recent years, the B-polynomials have attracted the attention of many researchers.These polynomials have been utilized for solving different equations by 
using various approximate methods.B-polynomials have been used for solving Fredholm integral equations [23] [24], Volterra's integral equations [25], Volterra'sFredholm-Hammerstein integral equations [26], differential equations [10] [27] [28] [29], integro-differential equations [30], parabolic equation subject to specification of mass[9] and so on.Singh et al. [27] and [10] have proposed operational matrices in different ways for solving differential equations.In [27], the B-polynomials have been firstly orthonormalized using Gram-Schmidt orthonormalization process and then the operational matrix of integration has been obtained. By the expansion of Bpolynomials in terms of Taylor basis, Yousefi and Behroozifar [10] have found the operational matrices of differentiation, integration and product of B-polynomials.

\subsection{Properties of B-polynomials}

\subsubsection{B-polynomials:}

As we mentioned, $m$-degree B-polynomials [29] are a set of polynomials defined on $[0,1]$ :

$$
B_{i, m}(x)=\left(\begin{array}{c}
m \\
i
\end{array}\right) x^{i}(1-x)^{m-i}, \quad 0 \leq i \leq m,
$$

where $\left(\begin{array}{c}m \\ i\end{array}\right)$ means

$$
\frac{m !}{i !(m-i) !},
$$

In this paper, we use the $\psi_{m}(x)$ notation to show

$$
\psi_{m}(x)=\left[\begin{array}{llll}
B_{0, m}(x) & B_{1, m}(x) & \cdots & B_{m, m}(x)
\end{array}\right]^{T},
$$

We should remember that [10]

$$
\psi_{m}(x)=A_{m} \times T_{m}(x),
$$

where

$$
T_{m}(x)=\left[x^{0} x^{1} \cdots x^{m}\right]^{T},
$$

and the $(i+1)^{\text {th }}$ row of matrix $A$ is

$$
A_{i+1}=\left[\begin{array}{lllllll}
\overbrace{0}^{\frac{i \text { times }}{0}} & \cdots & 0 & s_{0, i, m} & s_{1, i, m} & \cdots & s_{m, i, m}
\end{array}\right]
$$

where

$$
s_{j, i, m}=(-1)^{j}\left(\begin{array}{c}
m \\
i
\end{array}\right)\left(\begin{array}{c}
m-i \\
j
\end{array}\right)
$$

Matrix $A$ is an upper triangular matrix and

$$
\operatorname{det}(A)=\prod_{i=0}^{m}\left(\begin{array}{c}
m \\
i
\end{array}\right) ;
$$

so, $A$ is an invertible matrix. Then, we can obtain $A^{-1}$ using the following formula 


$$
\left\{A^{-1}\right\}_{i, j=0}^{m}= \begin{cases}\frac{\left(\begin{array}{c}
m-i \\
j-i
\end{array}\right)}{\left(\begin{array}{c}
m \\
j
\end{array}\right)}, & j \geq i \\
0, & j<i\end{cases}
$$

Now, to overcome the challenges of EOM approach, we should firstly introduce some relations and matrices.

\section{$2.3 \quad$ EOM-related relations}

\subsubsection{Recursive relation to differentiate B-polynomials:}

A known relation about the differentiation of B-polynomials is

$$
\begin{array}{rlr}
\frac{d}{d x} B_{i, m}(x) & =\left(\begin{array}{c}
m \\
i
\end{array}\right) i x^{i-1}(1-x)^{m-i}-\left(\begin{array}{c}
m \\
i
\end{array}\right)(m-i) x^{i}(1-x)^{m-i-1}, \\
& =m\left(\begin{array}{c}
m-1 \\
i-1
\end{array}\right) x^{i-1}(1-x)^{m-i}-m\left(\begin{array}{c}
m-1 \\
i
\end{array}\right) x^{i}(1-x)^{m-i-1}, \\
& =m\left(B_{i-1, m-1}(x)-B_{i, m-1}(x)\right), & 0 \leq i \leq m .
\end{array}
$$

\subsubsection{Finding the coordinate vector of a polynomial with respect to the basis:}

We aim to find a vector $b=\left[\begin{array}{llll}b_{0} & b_{1} & \cdots & b_{m}\end{array}\right]^{T}$ to be the Bernstein coordinate vector of the arbitrary polynomial $\sum_{i=0}^{m} c_{i} x^{i}$.

$$
\begin{aligned}
& \sum_{i=0}^{m} b_{i}\left(\begin{array}{c}
m \\
i
\end{array}\right) x^{i}(1-x)^{m-i}=\sum_{i=0}^{m} c_{i} x^{i} \\
& b^{T} \psi_{m}(x)=c^{T} T_{m}(x) .
\end{aligned}
$$

with respect to the matrix $A_{m \times m}$ in (1), we can write

$$
b^{T} A_{m \times m} T_{m}(x)=c^{T} T_{m}(x),
$$

So, we have

$$
b=\left(A^{-1}\right)^{T} c
$$

As an immediate result, we can propose a general formula for $x^{i}$ as follows:

$$
\begin{aligned}
& x^{i}=d_{i, m}^{T} \psi_{m}(x), \quad m \geq i,
\end{aligned}
$$

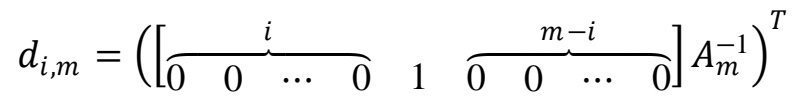

\subsection{EOM-related matrices}




\subsubsection{Working with $\mathrm{K}$ matrices:}

Firstly, we introduce a simple and useful matrix which we name it K-matrix:

$$
\begin{gathered}
K_{m, i}=\left[\begin{array}{ll}
I_{m} & 0_{m \times i}
\end{array}\right]_{m \times(m+i)} \\
K_{m, i}^{\prime}=\left[\begin{array}{ll}
0_{m \times i} & I_{-} m
\end{array}\right]_{m \times(m+i)}
\end{gathered}
$$

We can embed $i$ zero columns at the right and the left end of arbitrary $Z_{m \times m}$, using $Z \times K_{m, i}$ and $Z \times K_{m, i}^{\prime}$, respectively and $i$ zero rows at the bottom and top of the arbitrary $Z_{m \times m}$, using $K_{m, i}^{T} \times Z$ and $K_{m, i}^{\prime T} \times Z$, respectively.

\subsubsection{The increaser matrix:}

To obtain the so-called EOMs, first of all, we need to transform $\psi_{m}(x)$ to $\psi_{m+i}(x)$ and to find a matrix, $E_{m, m+i}$, such that

$$
\psi_{m}(x)=E_{m, i} \psi_{m+i}(x) .
$$

Using (1) and (8), we can write:

$$
\begin{aligned}
& A_{m} T_{m}(x)=E_{m, i} A_{m+i} T_{m+i}(x), \\
& A_{m} K_{m+1, i} T_{m+i}(x)=E_{m, i} A_{m+i} T_{m+i}(x)^{\circ}
\end{aligned}
$$

$K_{m+1, i} T_{m+i}(x)=T_{m}(x)$. This entails the following:

$$
E_{m, i}=A_{m} K_{m+1, i} A_{m+i}^{-1} .
$$

The size of matrix $E_{m, i}$ is $(m+1) \times(m+1+i)$. By using Equations(3), (4) and (8), we have

$$
\left\{\boldsymbol{E}_{m, i}\right\}_{p=0, q=0}^{m, m+i}= \begin{cases}\frac{\left(\begin{array}{c}
m \\
p
\end{array}\right)}{\left(\begin{array}{c}
m+i \\
q
\end{array}\right)} \sum_{j=0}^{q-p}(-1)^{j}\left(\begin{array}{c}
n-p \\
j
\end{array}\right)\left(\begin{array}{c}
n+i-p-j \\
q-p-j
\end{array}\right), & j \geq i \\
0, & j<i\end{cases}
$$

Now, we can increase the size of the Bernstein basis vector, using $E_{m, i}$ as follows

$$
\psi_{m}(x)=E_{m, i} \psi_{m+i}(x) .
$$

For example, if $m=3$ and $i=2$, then

$$
E_{3,2}=\left[\begin{array}{cccccc}
1 & \frac{2}{5} & \frac{1}{10} & 0 & 0 & 0 \\
0 & \frac{3}{5} & \frac{3}{5} & \frac{3}{10} & 0 & 0 \\
0 & 0 & \frac{3}{10} & \frac{3}{5} & \frac{3}{5} & 0 \\
0 & 0 & 0 & \frac{1}{10} & \frac{2}{5} & 1
\end{array}\right]
$$

\subsubsection{The differentiation matrix:}

Here, we intend to find a matrix to differentiate $\psi_{m}(x)$. Using (5), we have: 


$$
\frac{d}{d x} \psi_{m(x)}=m\left(\left[\begin{array}{c}
0 \\
\psi_{m-1}(x)
\end{array}\right]-\left[\begin{array}{c}
\psi_{m-1}(x) \\
0
\end{array}\right]\right),
$$

Now, using (8) and (8)

, we can write

$$
\begin{aligned}
& \frac{d}{d x} \psi_{m}(x)=m\left(\left(K^{\prime}\right)_{m, 1}^{T}-K_{m, 1}^{T}\right) \psi_{m-1}(x), \\
& \frac{d}{d x} \psi_{m}(x)=D_{m} \psi_{m-1}(x),
\end{aligned}
$$

So, we obtain the following

$$
D_{m}=m\left(\left(K^{\prime}\right)_{m, 1}^{T}-K_{m, 1}^{T}\right),
$$

\subsubsection{The integration Matrix:}

Considering (5), we have

$$
\begin{array}{lr}
\frac{d}{d x} B_{i, m}(x)=m\left(B_{i-1, m-1}(x)-B_{i, m-1}(x)\right), & 0 \leq i \leq m, \\
B_{i, m}(x)=m\left(\int_{0}^{x} B_{i-1, m-1}(x) d x-\int_{0}^{x} B_{i, m-1}(x) d x\right)+\left(\begin{array}{c}
m \\
i
\end{array}\right) 0^{i}, 0 \leq i \leq m,
\end{array}
$$

Using (13), while summing two consecutive B-polynomials, the middle terms cancel each other out

$$
\begin{aligned}
B_{i, m}(x)+ & B_{i+1, m}(x) \\
= & m\left(\int_{0}^{x} B_{i-1, m-1}(x) d x-\int_{0}^{x} B_{i, m-1}(x) d x+\int_{0}^{x} B_{i, m-1}(x) d x\right) \\
& -m\left(\int_{0}^{x} B_{i+1, m-1}(x) d x+\left(\begin{array}{c}
m \\
i
\end{array}\right) 0^{i}+\left(\begin{array}{c}
m \\
i+1
\end{array}\right) 0^{i+1}\right) \\
= & m\left(\int_{0}^{x} B_{i-1, m-1}(x) d x-\int_{0}^{x} B_{i+1, m-1}(x) d x+\left(\begin{array}{c}
m \\
i
\end{array}\right) 0^{i}\right), \\
& 0 \leq i \leq m .
\end{aligned}
$$

To sum more than two consecutive B-polynomials, we can follow up the same procedure

$$
\begin{aligned}
\sum_{k=i}^{m} B_{k, m}(x) & =m\left(\int_{0}^{x} B_{i-1, m-1}(x) d x-\int_{0}^{x} B_{m, m-1}(x) d x+\left(\begin{array}{c}
m \\
i
\end{array}\right) 0^{i}+\sum_{k=i+1}^{m}\left(\begin{array}{c}
m \\
k
\end{array}\right) 0^{k}\right) \\
& =m\left(\int_{0}^{x} B_{i-1, m-1}(x) d x-\int_{0}^{x} B_{m, m-1}(x) d x+\left(\begin{array}{c}
m \\
i
\end{array}\right) 0^{i}\right),
\end{aligned}
$$

$B_{m, m-1}(x)=B_{-1, m-1}(x)=0$; So, we have 


$$
\begin{aligned}
& \sum_{k=i}^{m} B_{k, m}(x)=m\left(\int_{0}^{x} B_{i-1, m-1}(x) d x+\left(\begin{array}{c}
m \\
i
\end{array}\right) 0^{i}\right), \quad 0 \leq i \leq m, \\
& \int_{0}^{x} B_{i, m}(x) d x=\frac{1}{m+1} \sum_{k=i+1}^{m+1} B_{k, m+1}(x)-\left(\begin{array}{c}
m \\
i+1
\end{array}\right) 0^{i+1}, 0 \leq i \leq m, \\
& \int_{0}^{x} B_{i, m}(x) d x=p_{i, m}^{T} \psi_{m+1}(x),
\end{aligned}
$$

where

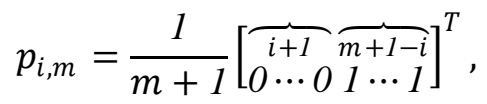

So,

$$
\int_{0}^{x} \psi_{m}(x) d x=P_{m} \psi_{m+1}(x)
$$

where

$$
P_{m}=\left[p_{0, m} \cdots p_{m, m}\right]_{(m+1) \times(m+2)}^{T} .
$$

\subsubsection{The product matrix:}

Here, we will describe the following property of the product of two B-Polynomials:

$$
\begin{aligned}
B_{i, m}(x) B_{j, n}(x) & =\left(\begin{array}{c}
m \\
i
\end{array}\right)\left(\begin{array}{c}
n \\
j
\end{array}\right) x^{i+j}(1-x)^{m+n-(i+j)}, \\
& =a_{i, j, m, n} B_{i+j, m+n}(x),
\end{aligned}
$$

where

$$
a_{i, j, m, n}=\frac{\left(\begin{array}{c}
m \\
i
\end{array}\right)\left(\begin{array}{c}
n \\
j
\end{array}\right)}{\left(\begin{array}{c}
m+n \\
i+j
\end{array}\right)}
$$

By using (15), we have:

$$
\begin{aligned}
& \psi_{m}(x) \psi_{n}^{T}(x) \\
& =\left[\begin{array}{ccccc}
a_{0,0, m, n} B_{0, m+n}(x) & \cdots & a_{0, j, m, n} B_{j, m+n}(x) & \cdots & a_{0, n, m, n} B_{n, m+n}(x) \\
\vdots & \ddots & \vdots & \ddots & \vdots \\
a_{i, 0, m, n} B_{i, m+n}(x) & \cdots & a_{i, j, m} B_{i+j, m+n}(x) & \cdots & a_{i, n, m, n} B_{i+n, m+n}(x) \\
\vdots & \ddots & \vdots & \ddots & \vdots \\
a_{m, 0, m, n} B_{m, m+n}(x) & \cdots & a_{m, j, m, n} B_{m+j, m+n}(x) & \cdots & a_{m, n, m, n} B_{m+n, m+n}(x)
\end{array}\right] .
\end{aligned}
$$

Consequently, for an arbitrary vector $c$, we can write:

$$
c^{T} \psi_{m}(x) \psi_{n}^{T}(x)=\psi_{m+n}^{T}(x) \times \tilde{C}_{m, n},
$$

where $\tilde{C}_{m, n}$ is an $(m+n+1) \times(n+1)$ product operational matrix for the vector $c$ 


$$
\tilde{C}_{m, n}=\left[\begin{array}{cccccc}
c_{0} a_{0,0, m, n} & 0 & \cdots & \cdots & \cdots & 0 \\
c_{1} a_{1,0, m, n} & c_{0} a_{0,1, m, n} & \ddots & \ddots & \ddots & \vdots \\
\vdots & \ddots & \ddots & \ddots & \ddots & \vdots \\
c_{j} a_{j, 0, m, n} & c_{j-1} a_{j-1,1, m, n} & \ddots & c_{0} a_{0, j, m, n} & \ddots & \vdots \\
\vdots & \ddots & \ddots & \ddots & \ddots & 0 \\
c_{m} a_{m, 0, m, n} & c_{m-1} a_{m 1,1, m, n} & \ddots & c_{m-j+1} a_{m-j+1, j, m, n} & \ddots & c_{0} a_{0, n, m, n} \\
0 & c_{m} a_{m, 1, m, n} & \ddots & c_{m-j+2} a_{m-j+2, j, m, n} & \ddots & c_{1} a_{1, n, m, n} \\
\vdots & \ddots & \ddots & \ddots & \ddots & \vdots \\
\vdots & \ddots & \ddots & c_{m} a_{m, j, m, n} & \ddots & c_{j-1} a_{j-1, n, m, n} \\
\vdots & \ddots & \ddots & \ddots & \ddots & \vdots \\
0 & \cdots & \cdots & \cdots & 0 & c_{m} a_{m, n, m, n}
\end{array}\right]
$$

or

$$
\left[\tilde{C}_{m, n}\right]_{i, j}=\left\{\begin{array}{lc}
0, & i \notin[j, j+m] \\
c_{i-j} a_{(i-j),(j-1), m, n}, & o . w
\end{array},\right.
$$

Also, by transposing (16), we have:

$$
\begin{aligned}
& \psi_{n}(x) \psi_{m}^{T}(x) c=\widehat{C_{n, m}} \psi_{m+n}(x), \\
& \widehat{C_{n, m}}=\tilde{C}_{m, n}^{T},
\end{aligned}
$$

\subsubsection{The power matrix:}

Suppose that $y(x)=c^{T} \psi_{m}(x)$, we aim to introduce $\overline{C_{m, n}}$ operational matrix by which

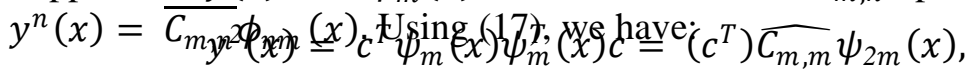

$$
y^{3}(x)=c^{T} \widehat{C_{m, m}} \psi_{2 m}(x) \psi_{m}^{T}(x) c=c^{T} \widehat{C_{m, m}} \widehat{C_{2 m, m}} \psi_{3 m}(x),
$$

Also, a similär result will be obtained for $y^{n}(x)$

$$
\begin{array}{rlr}
y^{n}(x) & =c^{T} \prod_{i=1}^{n-1} \widehat{C_{i m, m}} \psi_{n m}(x) n \geq 2, \\
& =\overline{C_{m, n}} \psi_{n m}(x), \quad n \geq 2
\end{array}
$$

where

$$
\overline{C_{m, n}}=c^{T} \prod_{i=1}^{n-1} \widehat{C_{i m, m}}, n \geq 2 .
$$

We name $\overline{C_{m, n}}$ the Bernstein power operational matrix.

\subsubsection{The Q Matrix:}

Suppose that we want to solve $\mathcal{N}[u(x)]=0$. Firstly, we choose an $m$ value by which the approximated $u(x)$ would be

$$
\begin{aligned}
& u(x) \approx y(x)=\sum_{i=0}^{m} c_{i} B_{i, m}(x)=c^{T} \psi_{m}(x), \\
& c=\left[\begin{array}{lll}
c_{0} c_{1} & \cdots & c_{m}
\end{array}\right]^{T},
\end{aligned}
$$

where the coefficients $c_{i}$ s are unknown.Then, we substitute the operations in $\mathcal{N}[y(x)]$ by the exact operational matrices to reach the exact residual function $\mathcal{N}\left[c^{T} \psi_{m}(x)\right]$; nevertheless, if the equation possesses some functions which does not exist in 


$$
Y_{m}=\operatorname{Span}\left(B_{0, m}(x), B_{1, m}(x), \cdots, B_{m, m}(x)\right),
$$

then, it would not be possible to have an exact residual function, which is because of the approach nature. However, we can write the new (exact) residual function as $R_{1 \times N} . \psi_{N}(x)$. We aim to set $c_{i}$ s so that the obtained residual function becomes as near as possible to zero. In this research, our interpretation of "best residual function" or "the nearest residual function to zero" for a specific defined norm \|| $\|$ is the function $r(x)$ for which

$$
\forall f(x) \in \operatorname{Span}\left(F_{m}(x)\right):\|r(x)\| \leq\|f(x)-r(x)\|
$$

In other words, we can say, $r(x)$ is a function which, its best approximation in $\operatorname{Span}\left(F_{m}(x)\right)$ is the zero function. To do so, firstly, consider the following theorem

\section{Theorem 2.1}

Consider the inner product space $H=L^{2}[a, b]$ and one of its finite-dimensional subspaces

$$
F_{m}=\operatorname{Span}\left(f_{0}(x), f_{1}(x), \cdots, f_{m}(x)\right),
$$

with the inner product defined by

$$
\langle f(t), g(t)\rangle=\int_{a}^{b} f(t) g(t) d t .
$$

If $p(x) \in F_{m}$ is the best approximation of $q(x) \in H$, then

$$
\langle q(x)-p(x), f\rangle=0,
$$

where

$$
\begin{aligned}
& f=\left[f_{0}(x) f_{1}(x) \cdots f_{m}(x)\right]^{T}, \\
& \langle f(x), f\rangle=\left[\left\langle f(x), f_{0}(x)\right\rangle\left\langle f(x), f_{1}(x)\right\rangle \cdots\left\langle f(x), f_{m}(x)\right\rangle\right]
\end{aligned}
$$

\section{Proof.}

A complete proof is given in [10]

Consider two inner product spaces $H=L^{2}[0,1]$ and $Y_{m}$ with the inner product defined by

$$
\langle f(t), g(t)\rangle=\int_{0}^{1} f(t) g(t) d t .
$$

We want the best approximation of $\operatorname{Residual}(x), \operatorname{in} Y_{m}$, to be zero. Using Theorem 2.1, we have

$$
\begin{aligned}
& \left\langle(\operatorname{Residual}(x)-0), \psi_{m}(x)\right\rangle=0, \\
& \left\langle\left(R_{1 \times(N+1)} \times \psi_{N}(x)-0\right), \psi_{m}(x)\right\rangle=0, N \geq m, \\
& R_{1 \times(N+1)} \times\left\langle\psi_{N}(x), \psi_{m}(x)\right\rangle=0, \quad N \geq m,
\end{aligned}
$$

therefore,

$$
R_{1 \times(N+1)} \times Q(N, m)=0,
$$

where $Q(N, m)$ is an $(N+1) \times(m+1)$ matrix 


$$
Q(N, m)=\left[q_{i j}\right]_{N+1 \times m+1}, q_{i j}=\left\langle B_{i-1, N}(x), B_{j-1, m}(x)\right\rangle, i \leq N, j \leq m,
$$

then, using (21), we solve

$$
R_{m}^{*}=0
$$

where

$$
R_{m}^{*}=R_{1 \times(N+1)} \times Q(N, m) .
$$

Also, we know that

$$
\begin{aligned}
q_{i j} & =\left\langle B_{i-1, N}(x), B_{j-1, m}(x)\right\rangle, \\
& \left.=\int_{0}^{1} \llbracket\left(\begin{array}{c}
N \\
i-1
\end{array}\right)\left(\begin{array}{c}
m \\
j-1
\end{array}\right) x^{i+j-2}(1-x)^{N+m-i-j+2} d x\right), \\
& =\frac{\left(\begin{array}{c}
N \\
i-1
\end{array}\right)\left(\begin{array}{c}
m \\
j-1
\end{array}\right)(N+m+2-(i+j)) !+(i+j-2) !}{N+m+1} .
\end{aligned}
$$

So, we have found the matrix $Q(N, m)=\left[q_{i j}\right]_{N+1 \times m+1}$, by which the $m$ final equations $\left(R_{m}^{*}\right)$ are obtained. By solving the obtained algebraic system, we will find $m+1$ unknown coefficients (elements of vector $c_{m}$ ) and, finally, find the $y(x) \simeq c_{m}^{T} \times \psi_{m}(x)$.

It is worth mentioning that the logic behind of the $Q$ matrix is exactly the logic of the Galerkin method; so, both of the results are the same and we call the $Q$ matrix as the Galerkin matrix.

\section{The error bound of the solution}

Before discussing about error bound of the solution, we describe the notation used in this section in brief. Suppose that $\mathcal{H}=C(\Gamma)$ denote the set of continuous functions in a linear space on a given closed interval $\Gamma=[a, b]$. A norm in $C[a, b]$, for all $f \in C[a, b]$, can be defined by

$$
\|f\|_{\infty}=\max _{a \leq x \leq b}|f(x)| .
$$

Let $\left\{x^{0}, x^{1}, x^{2}, \ldots, x^{n}\right\} \subset \mathcal{H}$ and suppose that

$$
\boldsymbol{J}=\operatorname{Span}\left(x^{0}, x^{1}, x^{2}, \ldots, x^{n}\right),
$$

be the set of all polynomials of degree at most $n$. Since $\mathcal{H}$ is Hilbert space and $\boldsymbol{J}$ is the finite-dimensional subspace, $\operatorname{dim} \boldsymbol{J}=n+1$, so $\boldsymbol{J}$ is a closed subspace of $\mathcal{H}$; therefore, $J$ is a complete subspace of $\mathcal{H}$ [31].

Let $f$ be an arbitrary element in $\mathcal{H}$, therefore, $f$ has a unique best approximation from $J$, say $\hat{\jmath} \in J$, that is [31]

$$
\exists \hat{\jmath} \in J ;|f-\hat{\jmath}|_{\infty}=\inf _{\forall j \in J}|f-j|_{\infty},
$$

The Weierstrass Approximation Theorem is a famous theorem in mathematical analysis. It asserts that every continuous function defined on a closed interval can be uniformly approximated as closely as desired by a polynomial function [32]. 
Theorem 3.1(The Weierstrass Approximation Theorem)

Let $f \in \mathcal{H}$. For any $\epsilon>0$, there exists a polynomial function $p$ such that for all $x$ in $[a, b]$, we have

$$
\|f(x)-p(x)\|_{\infty}<\epsilon .
$$

Proof; Refer to[33]

By a linear substitution, the interval $[a, b]$ can be transformed into the unit interval $[0,1]$. Thus, the original statement of the theorem holds if and only if the theorem holds for every continuous function $f$ defined on the interval $[0,1]$. Define the $n$th Bernstein polynomial for $f$ to be

$$
B_{m}(f ; x)=\sum_{k=0}^{m} f\left(\frac{k}{m}\right) B_{k, m}(x),
$$

\section{Theorem 3.2(The Bernstein Theorem)[34]}

Let $f$ be a continuous function defined on the closed interval[0,1]. For any $\epsilon>0$, there exists a positive integer $M$ such that for all $x$ in $[0,1]$ and integer $m \geq M$, we have

$$
\left\|f(x)-B_{m}(f ; x)\right\|_{\infty}<\epsilon .
$$

Remark 3.1; Note that the function $B_{m}(f ; x)$ is a polynomial on $x$. Thus, on the basis of the Bernstein Theorem, the Weierstrass Approximation Theorem holds.

Given a power-form polynomial $g$ of degree $n$, it is well known that for any $m \geq$ $n, g$ can be uniquely converted into a Bernstein polynomial of degree $m$ [35].

Now, given that the known function $f$ is $\mathrm{m}$ times continuously differentiable, the following lemma can be presented as an upper bound for estimating the error.

Lemma 3.1; Let the functiong : $[a, b] \rightarrow \mathbb{R}$ is $m+1$ times continuously differentiable, $g \in C^{m+1}[a, b]$, and

$$
Y=\operatorname{Span}\left(B_{0, m}(x), B_{1, m}(x), \ldots, B_{m, m}(x)\right) .
$$

If $c^{T} \psi_{m}(x)$ is the best approximation to $g$ from $Y$, then the mean error bound is presented as follows:

$$
\left\|g-c^{T} \psi_{m}(x)\right\|_{2} \leq \frac{M(b-a)^{\frac{2 m+3}{2}}}{(m+1) ! \sqrt{2 m+3}},
$$

where $M=\max _{a \leq x \leq b}=\left|g^{m+1}(x)\right|,\|f\|_{2}=\langle f, f\rangle^{\frac{1}{2}}$ and $\langle f, g\rangle=\int_{a}^{b} f(t) g(t) d t$.

Proof; A complete proof is given in [9]

Definition 3.1; Let $f(x)$ be defined on $[a, b]$, the modulus of continuity of $f(x)$ on $[a, b], w(\delta)$, is defined by

$$
w(\delta)=\sup _{|x-y|<\delta}|f(x)-f(y)|, \quad \text { for } \delta>0,
$$

We continue this section by stating two theorems concerned with estimating the error $f(x)-B_{n}(f ; x)$. The first one is the theorem of Elizaveta V. Voronovskaya (1898-1972), which gives an asymptotic error term for the Bernstein polynomials for functions that are twice differentiable.

There is the following result that gives an upper bound for the error $f(x)-B_{n}(f ; x)$ in terms of the modulus of continuity 
Theorem 3.3[36]

If $f$ is bounded on $[0,1]$, then

$$
\left\|f-B_{n}(f ; x)\right\|_{\infty} \leq \frac{3}{2} w\left(\frac{1}{\sqrt{n}}\right) .
$$

Theorem 3.4

If $f$ is bounded on $[0,1]$ and $Y=\operatorname{Span}\left\{B_{0, m}(x), B_{1, m}(x), \ldots, B_{m, m}(x)\right\}$, then

$$
\left\|f-c^{T} \psi_{m}(x)\right\|_{2} \leq \frac{3}{2} w\left(\frac{1}{\sqrt{m}}\right) .
$$

Where $c^{T} \psi_{m}(x)$ is the best approximation of $f$ in $Y$.

Proof;; A complete proof is given in [9]

Definition 3.2; Let $f(x) \in C[0,1]$ and $\phi:[0,1] \rightarrow \mathbb{R}$ be an admissible step-weight function (for details see [37]), the Ditzian-Totik modulus of smoothness of second order of $f(x)$ on $[0,1], w_{\phi}^{2}(\delta)$, is defined by

$$
\begin{array}{r}
w_{\phi}^{2}(\delta)=\sup _{|h| \leq \delta}\left(\sup _{x \pm h \phi(x) \in[0,1]}(|f(x-\phi(x) h)-2 f(x)+f(x+\phi(x) h)|)\right), \\
\text { for } \delta>0,
\end{array}
$$

\section{Theorem 3.5}

$\operatorname{Let} \varphi(x)=\sqrt{x(1-x)}$ and let $\phi:[0,1] \rightarrow \mathbb{R}, \phi \neq 0$ be an admissible step-weight function of the Ditzian-Totik modulus of smoothness such that $\phi^{2}$ and $\frac{\varphi^{2}}{\phi^{2}}$ are concave. Then, for any $f \in C[0,1]$ and $\lambda \in[0,1]$

$$
\left\|f(x)-B_{n}(f ; x)\right\|_{\infty} \leq C w_{\phi^{\lambda}}^{2}\left(\frac{1}{(\sqrt{n})} \varphi(x)^{1-\lambda}\right), \quad x \in[0,1] .
$$

Proof. A complete proof is given in [38]

Remark 3.2; The case $\lambda=0$ in (25) gives the classical local estimate [39], whereas $\lambda=1$ gives the global norm estimate developed by Ditzian and Totik [37].

Felten has,recently, showed the following theorem in [40]:

\section{Theorem 3.6}

Let $\varphi(x)=\sqrt{x(1-x)}$ and let $\phi:[0,1] \rightarrow \mathbb{R}, \phi \neq 0$ be an admissible step-weight function of the Ditzian-Totik modulus of smoothness such that $\phi^{2}$ and $\frac{\varphi^{2}}{\phi^{2}}$ are concave. Then, for $f \in C[0,1]$

$$
\left\|f(x)-B_{n}(f ; x)\right\|_{\infty} \leq C w_{\phi}^{2}\left(\frac{1}{\sqrt{n}} \frac{\varphi(x)}{\phi(x)}\right), \quad x \in[0,1] .
$$

Also for $\alpha \in(0,2)$, we have

$$
\left\|f(x)-B_{n}(f ; x)\right\|_{\infty} \leq C_{1}\left(\frac{1}{\sqrt{n}} \frac{\varphi(x)}{\phi(x)}\right)^{\alpha}, \quad x \in[0,1] .
$$

which implies that $w_{\phi}^{2}(\delta) \leq C_{2} \delta^{\alpha}$. 
Lemma 3.2; In Theorem 3.6 let the function $f:[a, b] \rightarrow \mathbb{R}$ to be $m+1$ times continuously differentiable, $\left(f \in C^{m+1}[a, b]\right)$ and

$$
Y=\operatorname{Span}\left\{B_{0, n}(x), B_{1, n}(x), \ldots, B_{n, n}(x)\right\} .
$$

If $c^{T} \psi_{n}(x)$ is the best approximation to $f$ in $Y$, then the mean error bound is presented as follows:

$$
\left\|f(x)-c^{T} \psi_{n}(x)\right\|_{2} \leq C w_{\phi}^{2}\left(\frac{1}{\sqrt{n}} \frac{\varphi(x)}{\phi(x)}\right), \quad x \in[0,1] .
$$

Proof; Sincec ${ }^{T} \psi_{n}(x)$ is the best approximation of $f$ from $Y$ and $B_{n}(f ; x) \in Y$, using $\|f\|_{2} \leq\|f\|_{\infty}$ we have

$$
\begin{aligned}
\left\|f(x)-c^{T} \psi_{n}(x)\right\|_{2} & \leq\left\|f(x)-B_{n}(f ; x)\right\|_{2} \\
& \leq\left\|f(x)-B_{n}(f ; x)\right\|_{\infty} \\
& \leq C w_{\phi}^{2}\left(\frac{1}{\sqrt{n}} \frac{\varphi(x)}{\phi(x)}\right), \quad x \in[0,1] .
\end{aligned}
$$

\section{Applications}

In this section, we compare the results obtained by EOMs with the results presented in [10] (in which OOMs for the Bernstein polynomials are introduced) to show the efficiency and accuracy of the present method.[10]includes two examples and some reports about them; but, to have a comprehensive comparison, we need some added reports.So, we implement OOMs as well as EOMs and run the codes together.

We compare the results in the first example with the exact solution and compute the norm 1 of the error and the residual function for each one.In the second example, the exact solution is not available except in special cases. Therefore, we only compare two methods residual functions.

We apply the present method to both examples for some increasing values of $m$ and report the following fraction formulas to study the convergence rate of both methods:

$$
\begin{aligned}
\text { Rate }_{1} & =\frac{\| \text { Residual }_{O O M}(x) \|_{1} \text { for the current } m}{\| \text { Residual }_{E O M}(x) \|_{1} \text { for the current } m}, \\
\text { Ratio }_{1}= & \frac{\| \text { Residual }_{E O M}(x) \|_{1} \text { for the previous } m}{\left\|\operatorname{Residual}_{E O M}(x)\right\|_{1} \text { for the current } m}, \\
\text { Rate }_{2}= & \frac{\left\|u(x)-y_{O O M}(x)\right\|_{1} \text { for the current } m}{\left\|u(x)-y_{E O M}(x)\right\|_{1} \text { for the current } m}, \\
\text { Ratio }_{2}= & \frac{\left\|u(x)-y_{E O M}(x)\right\|_{1} \text { for the previous } m}{\left\|u(x)-y_{E O M}(x)\right\|_{1} \text { for the current } m},
\end{aligned}
$$

The numerical implementation and all of the executions are performable by maplesoft.maple.16.x64, 64-bit Windows7 Ultimate Operating System, alongside hardware configuration: Laptop 64-bit Core i3 M380 CPU, 8 GBs of RAM.

\subsection{The Bessel differential equation}

Consider the following Bessel differential equation of order zero [41] 


$$
\mathcal{N}[u(x)]=0,
$$

where

$$
\mathcal{N}[\boldsymbol{u}(\boldsymbol{x})]=\boldsymbol{x} \boldsymbol{u}^{\prime \prime}(\boldsymbol{x})+\boldsymbol{u}^{\prime}(\boldsymbol{x})+\boldsymbol{x} \boldsymbol{u}(\boldsymbol{x})
$$

(Error! Bookmark not defined.)

with the initial conditions

$$
u(0)=1 \quad u^{\prime}(0)=0 .
$$

A solution known as the Bessel function of the first kind of order zero denoted by $J_{0}(x)$ is

$$
J_{0}(x)=\sum_{q=0}^{\infty} \frac{(-1)^{q}}{(q !)^{2}}\left(\frac{x}{2}\right)^{2 q} .
$$

Let $y^{\prime \prime}(x)$ to be an approximation of $u^{\prime \prime}(x)$ in $Y$ inner product space(19). Then

$$
\begin{aligned}
& y^{\prime \prime}(x)=c^{T} \psi_{m}(x), \\
& y^{\prime}(x)-y^{\prime}(0)=c^{T} P_{m} \psi_{m+1}(x),
\end{aligned}
$$

or

$$
\begin{aligned}
& y^{\prime}(x)=g^{T} \psi_{m+1}(x), \\
& g=P_{m}^{T} c .
\end{aligned}
$$

Also, using (7) we have

$$
\begin{aligned}
& y(x)-y(0)=g^{T} P_{m+1} \psi_{m+2}(x), \\
& y(x)=\left(g^{T} P_{m+1}+d_{0, m+2}^{T}\right) \psi_{m+2}(x)
\end{aligned}
$$

or

$$
\begin{aligned}
& y(x)=h^{T} \psi_{m+2}(x), \\
& h=P_{m+1}^{T} g+d_{0, m+2} .
\end{aligned}
$$

Also, using (26),(28),(7) and (17) we have

$$
\begin{aligned}
& x y^{\prime \prime}(x)=c^{T} \widehat{d_{1,1} m, 1} \psi_{m+1}(x) \\
& x y(x)=h^{T} \widehat{d_{1,1}}{ }_{m+2,1} \psi_{m+3}(x) .
\end{aligned}
$$

Now, consider the Eqs. (Error! Bookmark not defined.) and (11)

$$
\begin{aligned}
& \text { Residual }(x)=\mathcal{N}[y(x)] \\
& =\left(c^{T} \widehat{d_{1,1}}{ }_{m, 1}+g^{T}\right) E_{m+1,2} \psi_{m+3}(x)+h^{T} \widehat{d_{1,1}}{ }_{m+2,1} \psi_{m+3}(x) \\
& =\left(c^{T} \widehat{d_{1,1}, 1}+g^{T}\right) E_{m+1,2}+h^{T} \widehat{d_{1,1}(m+2,1)} \psi_{m+3}(x) \\
& =R_{(1 \times m+4)} \psi_{m+3}(x),
\end{aligned}
$$

where

$$
R=\left(c^{T} \widehat{d_{1,1} m, 1}+g^{T}\right) E_{m+1,2}+h^{T} \widehat{d_{1,1}}{ }_{m+2,1^{\prime}}
$$

Using (22), the only remaining issue is to solve the following system $(m+1$ equations and $m+1$ unknown elements of vector $c$ ) 


$$
R_{m}^{*}=0
$$

where

$$
R_{m}^{*}=R \times Q(m+3, m) .
$$
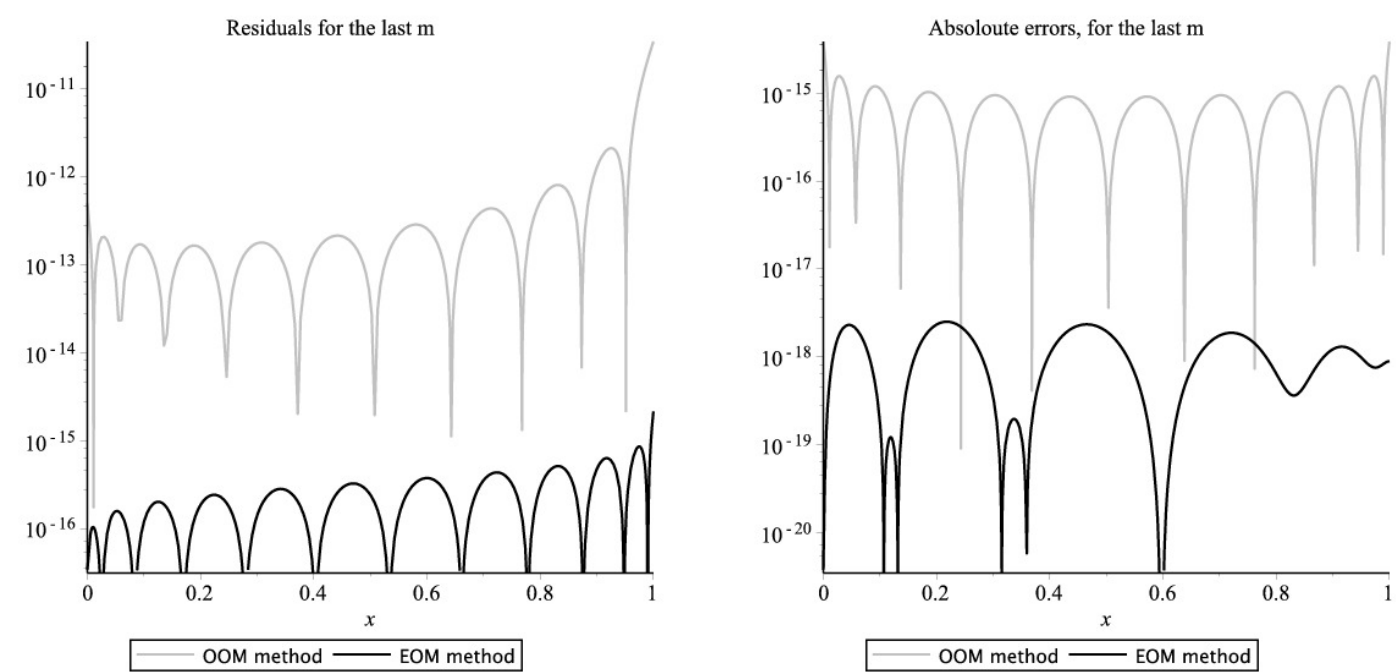

Figure 2

Graph of the absolute errors and Residual functions obtained by EOM and OOM for $\mathrm{m}=10$ in Example 1.
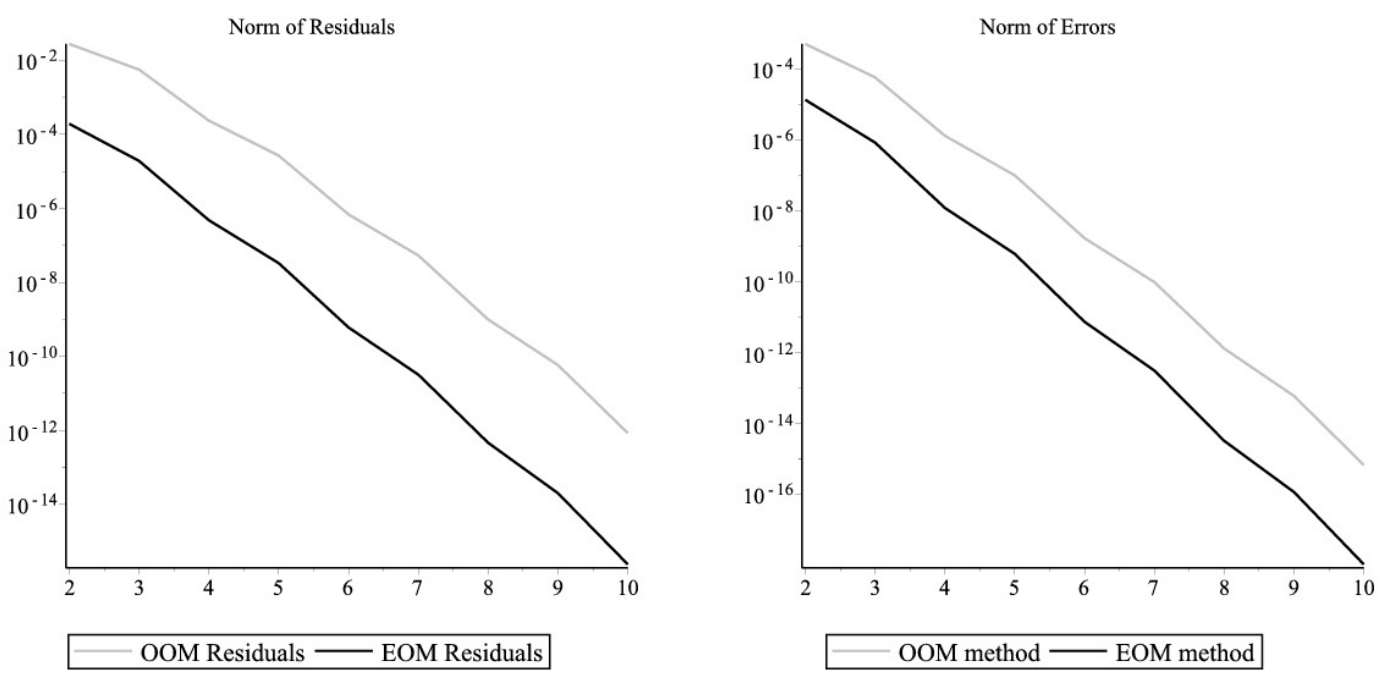

Figure 2

Graph of the absolute errors and Residual functions norms obtained by EOM and OOM in Example 1.

Eq. (29) is a system of nonlinear algebraic equations, which can be solved by a mathematical-software for the unknown elements of vector $c$.

Although, it is worth mentioning that it is too difficult to solve the systems of nonlinear equations even by Newton's method when the number of equation rises up; the main difficulty with such systems is how to choose the initial guess to handle the Newton method such that it results in low order computations. So, we applied the Galerkin method repeatedly. In the first step, we applied the Galerkin method for a small $n\left(\psi_{n}(x)\right)$ and set the initial guess to be a zero vector and gained a solution $y(x)=c_{1 \times n} \psi_{n}(x)$.Afterward, we applied the Galerkin method for a larger 
size ofbasis vector $\left(\psi_{n+i}(x)\right)$ and set the initial guess to be $c_{1 \times n} E_{n, i}$ which caused the solution of the last step to be the first guess of this step.

However, solving (29), the vector $c$ and, consequently, $y(x)=h^{T} \psi_{m+2}(x)$ is determined.The plots in Figure 2show the absolute error of both, $\operatorname{OOM}(\mid u(x)-$ $\left.y_{O O M}(x) \mid\right)$ and $\operatorname{EOM}\left(\left|u(x)-y_{E O M}(x)\right|\right)$ results and the Residualfunction of both methods for $m=10$. In Figure 2, the error functions norm $\left\|u(x)-y_{O o M}(x)\right\|_{1,[0,1]}$ and $\left\|u(x)-y_{E O M}(x)\right\|_{1,[0,1]},\|.\|_{1,[0,1]}=\int_{0}^{1}() d$.$x is reported.$

Also the norm of $\operatorname{Residual}(x)$ is shown for various values of $m$ for both EOM and OOM; which illustrates the convergence of both methods and the superiority of the present method.

In Table 1, a comparison is made between the absolute errors norm values, the residual functions norm values and the convergence rate of both EOM and OOM for those $m$ values which Yousefi [10]has reported.Table 2is a comparison of two methods for the values reported in Table (1) from [10].

Looking at Table 1,Table 2,Figure 2 and Figure 2, it can be observed that EOM provides much accurate results than OOM. They, also, show that the accuracy increases with the bases size growth.

\section{Table 1}

Comparison of some important numerical results for the Example 1.

\begin{tabular}{|c|l|c|c|c|}
\hline$m$ & $\|$ Res $_{\text {Oом }} \|_{1}$ & $\|$ Res $_{\text {EOM }} \|_{1}$ & Rate $_{1}$ & Ratio $_{1}$ \\
\hline 3 & 0.005633 & 0.00001919 & 293.6 & - \\
\hline 8 & $9.965 \mathrm{e}-10$ & $4.645 \mathrm{e}-13$ & 2146. & $4.131 \mathrm{e}-7$ \\
\hline 10 & $8.506 \mathrm{e}-13$ & $2.412 \mathrm{e}-16$ & 3526. & 1925. \\
\hline$m$ & $\|$ Err $_{\text {OOM }} \|_{1}$ & $\|$ Err $_{\text {EOM }} \|_{1}$ & Rate $_{2}$ & Ratio $_{2}$ \\
\hline 3 & 0.000057 & $8.282 \mathrm{e}-7$ & 68.82 & - \\
\hline 8 & $1.311 \mathrm{e}-12$ & $3.296 \mathrm{e}-15$ & 397.9 & $2.512 \mathrm{e} 8$ \\
\hline 10 & $6.880 \mathrm{e}-16$ & $1.093 \mathrm{e}-18$ & 629.6 & 3016. \\
\hline
\end{tabular}

\section{Table 2}

Comparison of OOM and EOM errors in various $\mathrm{x}$ values for the Example 1.

\begin{tabular}{|c|c|c|c|c|c|c|}
\hline \multirow{2}{*}{$x$} & \multicolumn{3}{|c|}{$m=3$} & \multicolumn{3}{c|}{$m=6$} \\
\cline { 2 - 7 } & OOM & EOM & Compare & OOM & EOM & Compare \\
\hline 0.0 & $0.1973 \mathrm{e}-3$ & 0 & $\infty$ & $0.6665 \mathrm{e}-11$ & 0 & $\infty$ \\
\hline 0.1 & $0.4839 \mathrm{e}-4$ & $0.7735 \mathrm{e}-6$ & 62.56 & $0.1152 \mathrm{e}-11$ & $0.4791 \mathrm{e}-14$ & 240.5 \\
\hline
\end{tabular}


K.Parand, S. A. Kaviani/ J. Math. Computer Sci. 6 (2013), 36- 59

\begin{tabular}{|c|c|c|c|c|c|c|}
\hline 0.2 & $0.7942 \mathrm{e}-4$ & $0.1555 e-5$ & 51.08 & $0.1601 \mathrm{e}-12$ & 0.8482e-15 & 188.8 \\
\hline 0.3 & $0.1811 \mathrm{e}-4$ & $0.1510 \mathrm{e}-5$ & 11.99 & $0.1378 \mathrm{e}-11$ & $0.7275 \mathrm{e}-14$ & 189.4 \\
\hline 0.4 & $0.5003 e-4$ & $0.9221 \mathrm{e}-6$ & 54.26 & 0.1590e-11 & 0.2097e-14 & 758.3 \\
\hline 0.5 & $0.7556 \mathrm{e}-4$ & $0.3819 \mathrm{e}-6$ & 197.8 & 0.1693e-12 & $0.9304 \mathrm{e}-15$ & 182.0 \\
\hline 0.6 & $0.4399 \mathrm{e}-4$ & $0.2825 \mathrm{e}-6$ & 155.7 & 0.1698e-11 & 0.6032e-14 & 281.5 \\
\hline 0.7 & $0.2552 \mathrm{e}-4$ & $0.6004 \mathrm{e}-6$ & 42.51 & $0.1144 \mathrm{e}-11$ & 0.2923e-14 & 391.2 \\
\hline 0.8 & $0.8186 \mathrm{e}-4$ & $0.9567 e-6$ & 85.56 & $0.4572 \mathrm{e}-12$ & $0.1632 \mathrm{e}-14$ & 280.2 \\
\hline 0.9 & $0.4384 \mathrm{e}-4$ & $0.9423 e-6$ & 46.52 & $0.1356 \mathrm{e}-11$ & 0.4018e-14 & 337.5 \\
\hline 1.0 & 0.1977e-3 & $0.6894 \mathrm{e}-6$ & 286.7 & $0.6664 \mathrm{e}-11$ & 0.2733e-14 & 2439. \\
\hline$x$ & \multicolumn{6}{|c|}{$m=9$} \\
\hline & \multicolumn{2}{|c|}{ OOM } & \multicolumn{2}{|c|}{ EOM } & \multicolumn{2}{|c|}{ Compare } \\
\hline 0.0 & \multicolumn{2}{|c|}{ 0.3831e-14 } & \multicolumn{2}{|c|}{0} & \multicolumn{2}{|l|}{$\infty$} \\
\hline 0.1 & \multicolumn{2}{|c|}{$0.1126 \mathrm{e}-14$} & \multicolumn{2}{|c|}{$0.1830 \mathrm{e}-18$} & \multicolumn{2}{|c|}{6150.} \\
\hline 0.2 & \multicolumn{2}{|c|}{ 0.9393e-15 } & \multicolumn{2}{|c|}{$0.2330 \mathrm{e}-17$} & \multicolumn{2}{|c|}{403.2} \\
\hline 0.3 & \multicolumn{2}{|c|}{$0.9421 \mathrm{e}-15$} & \multicolumn{2}{|c|}{$0.3510 \mathrm{e}-18$} & \multicolumn{2}{|c|}{2684.} \\
\hline 0.4 & \multicolumn{2}{|c|}{$0.6206 \mathrm{e}-15$} & \multicolumn{2}{|c|}{$0.1080 \mathrm{e}-17$} & \multicolumn{2}{|c|}{574.6} \\
\hline 0.5 & \multicolumn{2}{|c|}{$0.7229 \mathrm{e}-16$} & \multicolumn{2}{|c|}{ 0.1958e-17 } & \multicolumn{2}{|c|}{36.93} \\
\hline 0.6 & \multicolumn{2}{|c|}{$0.7176 \mathrm{e}-15$} & \multicolumn{2}{|c|}{$0.4117 \mathrm{e}-21$} & \multicolumn{2}{|c|}{0.1743 e 7} \\
\hline 0.7 & \multicolumn{2}{|c|}{$0.9424 \mathrm{e}-15$} & \multicolumn{2}{|c|}{ 0.1752e-17 } & \multicolumn{2}{|c|}{537.9} \\
\hline 0.8 & \multicolumn{2}{|c|}{ 0.8800e-15 } & \multicolumn{2}{|c|}{$0.6470 \mathrm{e}-18$} & \multicolumn{2}{|c|}{1360.} \\
\hline 0.9 & \multicolumn{2}{|c|}{$0.1084 \mathrm{e}-14$} & \multicolumn{2}{|c|}{$0.1221 \mathrm{e}-17$} & \multicolumn{2}{|c|}{887.5} \\
\hline 1.0 & \multicolumn{2}{|c|}{$0.3831 \mathrm{e}-14$} & 0.89 & $e-18$ & 4274 & \\
\hline
\end{tabular}

\subsection{The Emden-Fowler equation}

Consider the Emden-Fowler equation given in [42]

$$
u^{\prime \prime}(x)+\frac{2}{x} u^{\prime}(x)+x^{r} u^{n}(x)=0, \quad 0 \leq x \leq 1, \quad n \in \mathbb{N} \cup\{0\}
$$


with the initial conditions

$$
\begin{aligned}
& u(0)=1, \\
& u^{\prime}(0)=0 .
\end{aligned}
$$

For convenience, we can product both sides in $x$ and have

$$
\mathcal{N}[u(x)]=0,
$$

where

$$
\mathcal{N}[u(x)]=x u^{\prime \prime}(x)+2 u^{\prime}(x)+x^{r+1} u^{n}(x)
$$

Let $y^{\prime \prime}(s)$ be an approximation of $u^{\prime \prime}(s)$ in (31). We have

$$
\begin{aligned}
& y^{\prime \prime}(x)=c^{T} \psi_{m}(x), \\
& y^{\prime}(x)-y^{\prime}(0)=c^{T} P_{m} \psi_{m+1}(x),
\end{aligned}
$$

or

$$
\begin{aligned}
& y^{\prime}(x)=g^{T} \psi_{m+1}(x), \\
& g=P_{m}^{T} c .
\end{aligned}
$$

Also, using (7) we have

$$
\begin{aligned}
& y(x)-y(0)=g^{T} P_{m+1} \psi_{m+2}(x), \\
& y(x)=\left(g^{T} P_{m+1}+d_{0, m+2}^{T}\right) \psi_{m+2}(x)
\end{aligned}
$$

or

$$
\begin{aligned}
& y(x)=h^{T} \psi_{m+2}(x), \\
& h=P_{m+1}^{T} g+d_{0, m+2}
\end{aligned}
$$

So, using(18) and (34) we have

$$
y^{n}(x)=\overline{H_{m+2, n}} \psi_{(m+2) \cdot n}(x) .
$$

Also, using (32),(35),(7) and (17) we have

$$
\begin{aligned}
& x y^{\prime \prime}(x)=c^{T}\left(\widehat{d_{1,1}}\right) \psi_{m+1}(x) \\
& x^{r+1} y^{n}(x)=\overline{H_{m+2, n}}\left(\widehat{d_{r+1, r}+1}\right)_{m+2, r+1} \psi_{(m+2) \cdot n+r+1}(x) .
\end{aligned}
$$

Now, consider the Equations (31) and (11)

$$
\operatorname{Residual}(x)=R_{1 \times(m+2) \cdot n+r+2} \psi_{(m+2) \cdot n+r+1}(x),
$$

where

$$
R=\left(c^{T}\left(\widehat{d_{1,1}}\right)_{m, 1}+2 \cdot g^{T}\right) E_{m+1,(m+2) \cdot(n-1)+r+2}+\overline{H_{m+2, n}}\left(\widehat{d_{r+1, r+1}}\right)_{m+2, r+1}
$$

By using (22), the only remaining issue is to solve the following system $(m+1$ equations and $m+1$ unknown elements of vector $c$ ):

$$
R_{m}^{*}=0,
$$




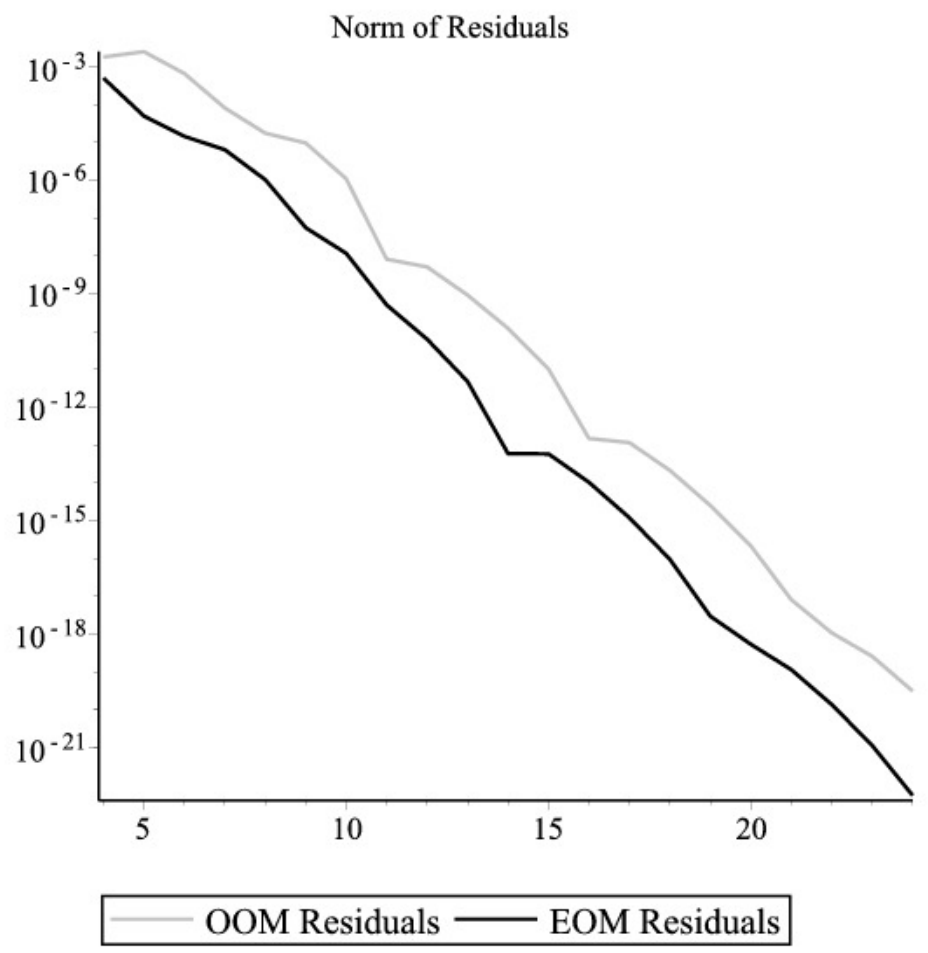

\section{Figure 3}

Graph of the absolute errors norms and residual functions norms for EOM and OOM in Example 2.

where,

$$
R_{m}^{*}=R \times Q((m+2) \cdot n+r+1, m) .
$$

Equation (37) is a system of nonlinear algebraic equations which its solution yields the vector $c$ elements. We solved the above equations system, setting $n=r=3$, for some increasing $m$ values.

We would like to state the numerical convergence, again, in the context of this example; therefore, in order to be assured of meaningful solutions, the numerical results are reported in Figure 3and Table 3, using residual functions norms. It is obviously observable that the residual functions norms tend to zero, while increasing the size of bases. As mentioned before, the analytic solution of $y(x)$ does not exist, thus we only compared the residual functions.

Table 3 is the numerical report of the information depicted in Figure 3,alongside, a comparison between the residual functions norm values related to both methods in the 3rd column and also the comparison between the convergence rates of EOM in each step to its previous step in the 4 th column.Paying attention to the table, it can be seen that the approximations are improved by increasing the size of the basis, which confirms the proposed methods convergence. Also the convergence rate of EOM seems to be greater than OOM which shows superiority of the present method.

\section{Table 3}

Comparison of numerical results for Example 2. 
K.Parand, S. A. Kaviani/ J. Math. Computer Sci. 6 (2013), 36- 59

\begin{tabular}{|c|c|c|c|c|}
\hline$m$ & $\left\|\operatorname{Residual}_{\text {OоM }}(x)\right\|_{1}$ & $\|$ Residual $_{E O M}(x) \|_{1}$ & Rate $_{1}$ & Ratio $_{1}$ \\
\hline 4 & $0.1781 \mathrm{e}-2$ & $0.4921 \mathrm{e}-3$ & 3.620 & - \\
\hline 5 & $0.2454 \mathrm{e}-2$ & $0.4891 \mathrm{e}-4$ & 50.18 & 10.06 \\
\hline 6 & $0.6502 \mathrm{e}-3$ & $0.1422 \mathrm{e}-4$ & 45.73 & 3.440 \\
\hline 7 & $0.7988 \mathrm{e}-4$ & $0.6298 \mathrm{e}-5$ & 12.68 & 2.257 \\
\hline 8 & $0.1725 \mathrm{e}-4$ & $0.1016 \mathrm{e}-5$ & 16.97 & 6.198 \\
\hline 9 & $0.9499 \mathrm{e}-5$ & $0.5474 \mathrm{e}-7$ & 173.5 & 18.56 \\
\hline 10 & $0.1077 e-5$ & $0.1153 e-7$ & 93.42 & 4.747 \\
\hline 11 & 0.8083e-8 & $0.4994 \mathrm{e}-9$ & 16.19 & 23.09 \\
\hline 12 & $0.5047 \mathrm{e}-8$ & $0.6202 \mathrm{e}-10$ & 81.37 & 8.051 \\
\hline 13 & $0.9102 \mathrm{e}-9$ & $0.4751 \mathrm{e}-11$ & 191.6 & 13.06 \\
\hline 14 & $0.1202 \mathrm{e}-9$ & $0.5882 \mathrm{e}-13$ & 2043. & 80.78 \\
\hline 15 & $0.1012 \mathrm{e}-10$ & $0.5800 \mathrm{e}-13$ & 174.4 & 1.014 \\
\hline 16 & $0.1486 \mathrm{e}-12$ & $0.1031 \mathrm{e}-13$ & 14.42 & 5.626 \\
\hline 17 & $0.1149 \mathrm{e}-12$ & 0.1192e-14 & 96.46 & 8.651 \\
\hline 18 & $0.2130 \mathrm{e}-13$ & $0.9520 \mathrm{e}-16$ & 223.8 & 12.52 \\
\hline 19 & $0.2515 \mathrm{e}-14$ & $0.2983 \mathrm{e}-17$ & 843.4 & 31.92 \\
\hline 20 & $0.2163 e-15$ & $0.5429 \mathrm{e}-18$ & 398.3 & 5.493 \\
\hline 21 & 0.8191e-17 & 0.1159e-18 & 70.68 & 4.685 \\
\hline 22 & $0.1086 \mathrm{e}-17$ & 0.1386e-19 & 78.36 & 8.364 \\
\hline 23 & $0.2605 \mathrm{e}-18$ & $0.1150 \mathrm{e}-20$ & 226.4 & 12.04 \\
\hline 24 & 0.3145e-19 & $0.5500 \mathrm{e}-22$ & 571.9 & 20.92 \\
\hline
\end{tabular}

\section{Concluding Remarks and Future Works}

Using operational matrices and also the Galerkin method are two techniques which each one, individually, is used for solving dynamical systems.In this paper an idea is presented by which the exact operational matrices are achievable; so, we can differentiate, integrate and product the vector(s) of basis functions (basis vector(s)) exactly. 
We implemented this idea on the Bernstein basis vector.Using such exact matrices and substituting them by equation operators including integration, differentiation and product, we reached the exact residual function of the equation, which can be written in the form $R . \phi(x)$, where $R$ is an algebraic equation vector and $\phi(x)$ is the basis vector.Then, we presented a matrix $Q$ for which $R . Q$ would be the output equations system of the Galerkin method.Also, we discussed about a technique,presented for finding some appropriate starting values, for solving the resulting nonlinear algebraic system which may be large and has a high computational complexity.

Afterward, we solved a linear and a nonlinear problem by using both of the exact and the ordinary operational matrices to prove the convergence of methods and also the presented method superiority.As some future works, higher-nonlinearity-degree problems can be solved by both methods to show the superiority of the new method much obviously.

\section{Acknowledgements}

We thank Mr. Zafarvahedian for making available to us his study results regarding the Bernstein polynomials

\section{References}

1. M. Razzaghi and M. Razzaghi, Fourier series direct method for variational problems, I. J. Control.48, 887895(1988)

2. M. Razzaghi and M. Razzaghi, Taylor series analysis of time-varying multi-delay systems, I. J. Control. 50, 183-192(1989)

3. M. Razzaghi and M. Razzaghi, Shifted-Jacobi series direct method for variational problems, Int. J. Syst. Sci. 20, 1119-1129(1989)

4. E. H. Doha andA. H. Bhrawy and S. S. Ezz-Eldien, A new Jacobi operational matrix: An application for solving fractional differential equations,Appl. Math. Model. 36, 4931-4943 (2012)

5. M. Razzaghi and S. A. Yousefi, Legendre wavelets direct method for variational problems,Math. Comput. Simulat.53, 185-192(2000)

6. S. A. Yousefi and H. Jafari and M. A. Firoozjaeeand S. Momani and C. M. Khaliqued, Application of Legendre wavelets for solving fractional differential equations, Comput. Math. Appl. 62, 1038-1045(2011)

7. S. A. Yousefi and F. Khellat,The linear Legendre mother wavelets operational matrix of integration and its application, J. Franklin I. 343, 181-190(2006)

8. S. A. Yousefi and M. Behroozifar and M. Dehghan, Numerical solution of the nonlinear age-structured population models by using the operational matrices of Bernstein polynomials, Appl. Math. Modell. 36, 945963 (2012)

9. S. A. Yousefi and M. Behroozifar and M. Dehghan, The operational matrices of Bernstein polynomials for solving the parabolic equation subject to specification of the mass, J. Comput. Appl. Math.335, 52725283(2011)

10. S. A. Yousefi and M. Behroozifar, Operational matrices of Bernstein polynomials and their applications, I. J. Sys. Sci. 41, 709-716(2010)

11. A. H. Bhrawy, The operational matrix of fractional integration for shifted Chebyshev polynomials, Appl. Math. Lett. 26, 25-31(2013)

12. H. R. Marzban and M. Razzaghi, Solution of multi-delay systems using hybrid of block-pulse functions and Taylor series, J. Sound Vib. 292, 954-963 (2006)

13. H. R. Marzban and M. Razzaghi, Optimal control of linear delay systems via hybrid of block-pulse and Legendre polynomials, J. Franklin I. 341, 279-293 (2004)

14. H. R. Marzban and M. Shahsiah, Solution of piecewise constant delay systems using hybrid of blockpulse and Chebyshev polynomials, Optim. Contr. Appl. Met., Vol. 32, pp. 647-659 (2011)

15. M. Lakestani and M. Dehghan and S. I. Pakchin, The construction of operational matrix of fractional derivatives using B-spline functions, Commun. Nonlinear. Sci. Numer. Simulat. 17, 1149-1162 (2012)

16. S. Kazem and M. Shaban and J. A. Rad, Solution of the coupled burgers equation based on operational matrices of d-dimensional orthogonal functions, Z. Naturforsch. A. 67, 267-274 (2012)

17. M. Shaban and S. Kazem and J. A. Rad, A modification of the homotopy analysis method based on Chebyshev operational matrices, Math. Comput. Model, in press(2013) 
18. S. Nemati and P. M. Lima and Y. Ordokhani, Numerical solution of a class of two-dimensional nonlinear Volterra integral equations using Legendre polynomials, J. Comput. Appl. Math. 242, 53-69 (2013)

19. F. Mohammadi and M. Hosseini, A new Legendre wavelet operational matrix of derivative and its applications in solving the singular ordinary differential equations, J. Franklin I.348, 1787-1796 (2011)

20. A. Saadatmandi and M. Dehghan, A new operational matrix for solving fractional-order, Comput. Math. Appl. 59, 1326-1336 (2010)

21. A. Saadatmandi and M. Dehghan, A tau approach for solution of the space fractional diffusion equation, Comput. Math. Appl. 62, 1135-1142 (2011)

22. E. W. Weisstein, Editor, CRC Concise Encyclopedia of Mathematics. $2^{\text {nd }}$,Chapman and Hall/CRC, Champaign (2002).

23. A. Chakrabarti and S. C. Martha, Approximate solutions of Fredholm integral equations of the second kind, Appl. Math. Comput. 211, 459-466 (2009)

24. B. N. Mandal, and S. Bhattacharya, Numerical solution of some classes of integral equations using Bernstein polynomials, Appl. Math. Comput. 190, 1707-1716 (2007)

25. S. Bhattacharya and B. N. Mandal, Use of Bernstein polynomials in numerical solutions of Volterra integral equations, Appl. Math. Sci. 2, 1773-1787(2008)

26. K. Maleknejad and E. Hashemizadeh and B. Basirat, Computational method based on Bernestein operational matrices for nonlinear Volterra-Fredholm-Hammerstein integral equations, Commun. Nonl. Sci. Numer. Simul. 17, 52-61(2012)

27. A. K. Singh and V. K. Singh andO. P. Singh, The Bernstein operational matrix of integration, Appl. Math. Sci. 3, 2427-2436 (2009)

28. M. I. Bhatti and D. D. Bhatta, Numerical solution of $\mathrm{KdV}$ equation using modified Bernstein polynomials, Appl. Math. Comput. 174, 1255-1268 (2006)

29. M. I. Bhatti and P. Bracken, Solutions of differential equations in a Bernstein polynomial basis, J. Comput. Appl. Math. 205, 272-280 (2007)

30. S. Bhattacharya andB. N. Mandal, Numerical solution of a singular integro-differential equation, Appl. Math. Comput.195, 346-350 (2008)

31. E. Kreyszig, Introductory functional analysis with applications, John Wiley, New York (1978)

32. P. J. Davis, Interpolation and Approximation, Blaisdell Publishing Company (1963)

33. G. Lorentz, Bernstein Polynomials, University of Toronto Press (1953)

34. K. M. Levasseur, A probabilistic proof of the Weierstrass approximation theorem, Am. Math. Mon. 4, 249-250(1984)

35. R. Farouki, V. Rajan, On the numerical condition of polynomials in Bernstein form, Comput. Aided Geom. D. 3, 191-216 (1987)

36. T. J. Rivlin, An Introduction to the Approximation of Functions, Dover publications (1981)

37. Z. Ditzian andV. Totik, Moduli of Smoothness, Springer-Verlag, New York(1987)

38. Z. Ditzian, Direct estimate for Bernstein polynomials, J. Approx. Theory 79, 165-166 (1994)

39. L. I. Strukov and A. F. Timan, Mathematical expectation of continuous functions of random variables, smoothness and variance, Sibreian Math. J. 18, 469-474(1977)

40. M. Felten, Direct and inverse estimates for Bernstein polynomials, Constr. Approx. 14, 459-468 (1998)

41. P. V. O'Neil, Advanced Engineering Mathematics. Wadsworth, Belmont (1987)

42. A. M. Wazwaz, Adomian Decomposition Method for a Reliable Treatment of the Emden-Fowler Equation, Appl. Math. Comput. 161, 543-560 (2005) 